\title{
Inventory, differentiation, and proportional diversity: a consistent terminology for quantifying species diversity
}

\author{
Gerald Jurasinski · Vroni Retzer · Carl Beierkuhnlein
}

Received: 18 December 2007/ Accepted: 13 August 2008/Published online: 25 October 2008

(C) Springer-Verlag 2008

\begin{abstract}
Almost half a century after Whittaker (Ecol Monogr 30:279-338, 1960) proposed his influential diversity concept, it is time for a critical reappraisal. Although the terms alpha, beta and gamma diversity introduced by Whittaker have become general textbook knowledge, the concept suffers from several drawbacks. First, alpha and gamma diversity share the same characteristics and are differentiated only by the scale at which they are applied. However, as scale is relative-depending on the organism(s) or ecosystems investigated-this is not a meaningful ecological criterion. Alpha and gamma diversity can instead be grouped together under the term "inventory diversity." Out of the three levels proposed by Whittaker, beta diversity is the one which receives the most contradictory comments regarding its usefulness ("key concept" vs. "abstruse concept"). Obviously beta diversity means different things to different people. Apart from the large variety of methods used to investigate it, the main reason for this may be different underlying data
\end{abstract}

Communicated by Diethart Matthies.

Electronic supplementary material The online version of this article (doi:10.1007/s00442-008-1190-z) contains supplementary material, which is available to authorized users.

\section{G. Jurasinski ( $\square)$}

Landscape Ecology and Site Evaluation,

Faculty of Agricultural and Environmental Sciences,

University of Rostock, Justus-von-Liebig-Weg 6,

18059 Rostock, Germany

e-mail: gerald.jurasinski@uni-rostock.de

URL: http://www.auf.uni-rostock.de/loe/

V. Retzer · C. Beierkuhnlein

Biogeography, University of Bayreuth,

95440 Bayreuth, Germany

URL: http://www.uni-bayreuth.de/departments/biogeo/ characteristics. A literature review reveals that the multitude of measures used to assess beta diversity can be sorted into two conceptually different groups. The first group directly takes species distinction into account and compares the similarity of sites (similarity indices, slope of the distance decay relationship, length of the ordination axis, and sum of squares of a species matrix). The second group relates species richness (or other summary diversity measures) of two (or more) different scales to each other (additive and multiplicative partitioning). Due to that important distinction, we suggest that beta diversity should be split into two levels, "differentiation diversity" (first group) and "proportional diversity" (second group). Thus, we propose to use the terms "inventory diversity" for within-sample diversity, "differentiation diversity" for compositional similarity between samples, and "proportional diversity" for the comparison of inventory diversity across spatial and temporal scales.

Keywords Beta diversity - Alpha diversity ·

Gamma diversity · Additive partitioning .

Compositional similarity

\section{Introduction}

"List(s) of the actual species [...] are needed, not estimates of beta-diversity, which can be estimated in many ways because no two ecologists can agree on what beta-diversity is!"

—Anonymous reviewer

This quote is taken from a review of one of our manuscripts. Although we do not agree with the statement, it stimulated us to carry out a thorough review of the different concepts behind beta diversity. Based on this, we discuss 
its various facets here and place them in a terminological context.

There are more ways to assess the diversity in species assemblages than just counting species numbers (e.g., Magurran 2003; Allan 1975). Peirce (1884), Jaccard (1901) and Williams (1950) all expressed the idea of additionally using the heterogeneity of samples as a measure of diversity. However, Whittaker $(1956,1960)$ was the first to develop a framework incorporating different aspects of biotic diversity. In his groundbreaking work on the vegetation of the Siskyou Mountains (Whittaker 1960, p. 620), he developed a terminology and concept for the measurement and comparison of vegetation diversity in which he distinguishes three aspects or levels of species diversity in natural communities: (1) alpha diversity, the "richness in species of a particular stand or community;" (2) beta diversity, the "extent of change of community composition;" (3) gamma diversity, the "species diversity of a number of community samples." Whittaker (1960) concludes that the "same types of measurements may be applied to 'gamma' as to 'alpha' diversity," whilst " 'beta' diversity represents a different problem."

The concept has found its way into ecological textbooks (e.g., Magurran 2003; Rosenzweig 1995; Beierkuhnlein 2006) and has been widely accepted. However, there is also debate over its usefulness-especially regarding beta diversity. Therefore, here we propose a critical appraisal of the concept that reflects the recent discussion and leads to a new terminology that clarifies the characteristics of different beta diversity measures.

Species richness and species frequencies (alpha diversity) have long been used as basic parameters for describing biotic diversity (e.g., Fisher 1943; Preston 1948; Hector and Bagchi 2007). Beta diversity has been used to test nichebased models against neutral models of species assembly (e.g., Ruokolainen et al. 1997; Condit et al. 2002; Tuomisto et al. 2003; Chust et al. 2006) and for the evaluation of distance decay at continental scales (Nekola and White 1999; Qian et al. 1998). To a smaller extent, the concept has also been employed in conservation-related studies (e.g., Steinitz et al. 2005; Wiersma and Urban 2005) or in studies on biological homogenization (e.g., McKinney 2004; Olden et al. 2006; Jurasinski and Kreyling 2007).

Recently, the importance of beta diversity in ecological research has found itself the subject of debate. There is substantial disagreement about biodiversity conceptualization and evaluation among ecologists (Ghilarov 1996; Ricotta 2005; Sarkar 2005; Sarkar 2006; Podani 2006). Legendre et al. (2005) emphasize that beta diversity is "a key concept for understanding the functioning of ecosystems, for the conservation of biodiversity, and for ecosystem management," because it can be used to describe the distribution of species diversity in space and/or time.
Many scientists call for a stronger incorporation of beta diversity into ecological research (e.g., Condit et al. 2002; Olden and Rooney 2006) and in conservation planning (e.g., Srivastava 2002; Wiersma and Urban 2005). Other authors regard beta diversity an "abstruse concept" (Novotny and Weiblen 2005), and it has become widely accepted that beta diversity has grown to incorporate a wide range of concepts. The multifaceted nature of beta diversity makes it difficult to completely describe it with a simple single definition, and its ambiguity has been criticized (see, e.g., Vellend 2001; Lorance et al. 2002; Ricotta 2005).

As a concept, beta diversity is not as singularly defined as alpha and gamma diversity. Maybe this is why a large variety of methods are available to investigate beta diversity. Comparisons between the results of different studies may be hindered severely by the variety of measures used to quantify beta diversity and by the variety of ways in which these measures are applied (Koleff et al. 2003a). However, the imprecision surrounding the term beta diversity is not only due to the multitude of available measures, but to the fact that these are related to different conceptual backgrounds which are not clearly explained and thoroughly understood. Whittaker (1960) laid the foundation for future confusion, as he proposed several different concepts of beta diversity. All are related to the idea that the heterogeneity of an ecosystem or a landscape can be examined based on the joint analysis of single observations within this ecosystem/landscape. Here, we give a short but comprehensive review of the different interpretations of Whittaker's terminological concept, placing a strong emphasis on beta diversity since this term causes the most confusion due to its many meanings. This compilation can provide a useful basis for future discussion and reference.

We show that the different concepts of beta diversity can be organized into two groups based on whether species distinction or species numbers are considered. Based on this review we develop and discuss an alternative terminology for the measurement and analysis of species diversity that clearly reflects the underlying data characteristics and different applications and will thus aid a better understanding of the different aspects of species diversity.

\section{Methods}

This paper is based on our own work on beta diversity issues (Beierkuhnlein 2000, 2001; Jurasinski and Beierkuhnlein 2006; Buhk et al. 2007; Jurasinski and Kreyling 2007), as well as on a review of recent and classic literature regarding beta diversity. To achieve a systematic overview of the usage of different beta diversity concepts in the literature, we conducted a search in the ISI "Web of Science," which 
represents one of the largest and most comprehensive databases of anglophone natural science literature on the web. All 75 ecological papers with "beta" and "diversity" or "diversities" in the title were screened manually. We skipped two papers from the analyses because one could not be accessed, and another one mentioned beta diversity solely in the title. The resulting 73 papers were analyzed systematically regarding the kind of beta diversity concept employed. The complete reference list of the analyzed papers including information on the type of study and beta diversity concept employed is available as "Electronic supplementary material."

Alpha, beta and gamma diversity and their development

\section{Alpha and gamma diversity}

As Whittaker (1960) noted, alpha and gamma diversity are descriptors of species within one certain area, but they differ in the units considered. Alpha diversity is measured within a sample (in Whittaker's original notion, a stand or community; other frequently used terms are: site, sampling unit, plot, etc.; in the following we use the term "sample"), while gamma diversity refers to the species richness at a higher aggregational level, usually a combination of different samples within the investigation area (Fig. 1). Whittaker (1956) introduced the term alpha diversity, as he suggested that Fisher's alpha parameter of the log-series species-abundance distribution would be a useful measure. There are basically two different types of measures for alpha and gamma diversity. The first is sampled species richness or estimated species richness, using samples or individual-based rarefaction (species accumulation curves, e.g., Gotelli and Colwell 2001; Chao 2005): the second describes the species-abundance distribution, such as the indices of Simpson (1949); Shannon-Weaver (1949) or Fisher's alpha (1943).

\section{The different notions of beta diversity}

Beta diversity is frequently used in a very general sense of differentiation between units (e.g., Condit et al. 2002; Koleff et al. 2003b; Chave 2004; Kluth and Bruelheide 2004; Chust et al. 2006; Olden and Rooney 2006). Vellend (2001) tried to clarify terminology by distinguishing between beta diversity (relationship between the species richness or representatives of species richness at different scale levels) and "species turnover" (compositional similarity). On the one hand, this might indicate that "species turnover" is different from beta diversity. On the other hand, several approaches to beta diversity are neglected. Alternatively, two main groups of beta diversity measures can be distinguished that represent different approaches to

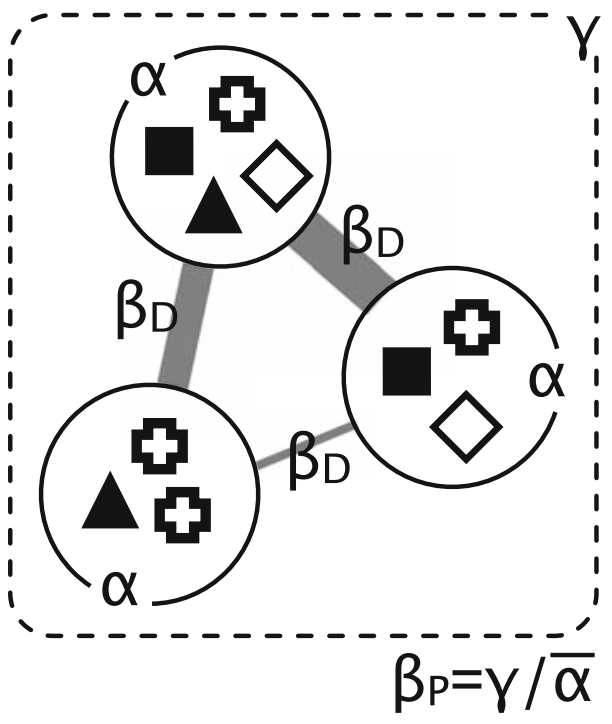

Fig. 1 Illustration of alpha, beta, and gamma diversity. The circles represent vegetation samples (relevés) with species (different symbols depict different species). The dashed box encloses the set of samples. The species found in this set represent the gamma diversity. For simplicity, alpha diversity (diversity within samples) is recorded as species richness. The same holds for gamma diversity (diversity within a set of samples or within a larger region). Beta cannot easily be illustrated due to its multifaceted character. The two main types of variety among samples are differentiation diversity $\left(\beta_{\mathrm{D}}\right)$, which is shown as similarity between pairs of samples taking into account species distinction (the broader the gray bar connecting two samples, the higher the similarity), and proportional diversity $\left(\beta_{\mathrm{P}}\right)$, which is assessed as the relation between the two scales of investigation (taking into account species numbers) in terms of species richness. $\beta_{\mathrm{P}} \neq \beta_{\mathrm{D}}$. For further explanation, see text

the handling of recorded information. One group comprises coefficients that examine the variation in species richness (expressed by species numbers or by richness indices) across scales. The other group comprises coefficients that examine the variation in species composition between samples (taking into account species distinction and/or abundances).

We tried to depict the two main concepts of beta that derive from this distinction and their relation to alpha and gamma diversity to illustrate the fundamental differences between them (Fig. 1). Various mathematical forms of beta diversity exist that can be assigned to these two concepts. However, they are not equally widespread in the screened literature, and only few approaches (e.g., resemblance indices, multiplicative partitioning) have been applied relatively often (Fig. 2).

\section{Concepts of beta diversity: Group 1-examining the variation in species richness}

Multiplicative partitioning In his original paper, Whittaker (1960) defined beta diversity as the relation between gamma and average alpha diversity: 
Fig. 2 Number of times the different concepts of beta diversity have been employed in the screened literature. The absolute number of cases the different concepts have been employed in the screened literature may give an impression of the overall frequencies of implementation in the ecological literature. The concept of resemblance [including all kinds of (dis)similarity and distance measures] is by far the most often applied. The numbers do not add up to the number of studies considered because some papers dealt with more than one concept
Implementation of different beta concepts

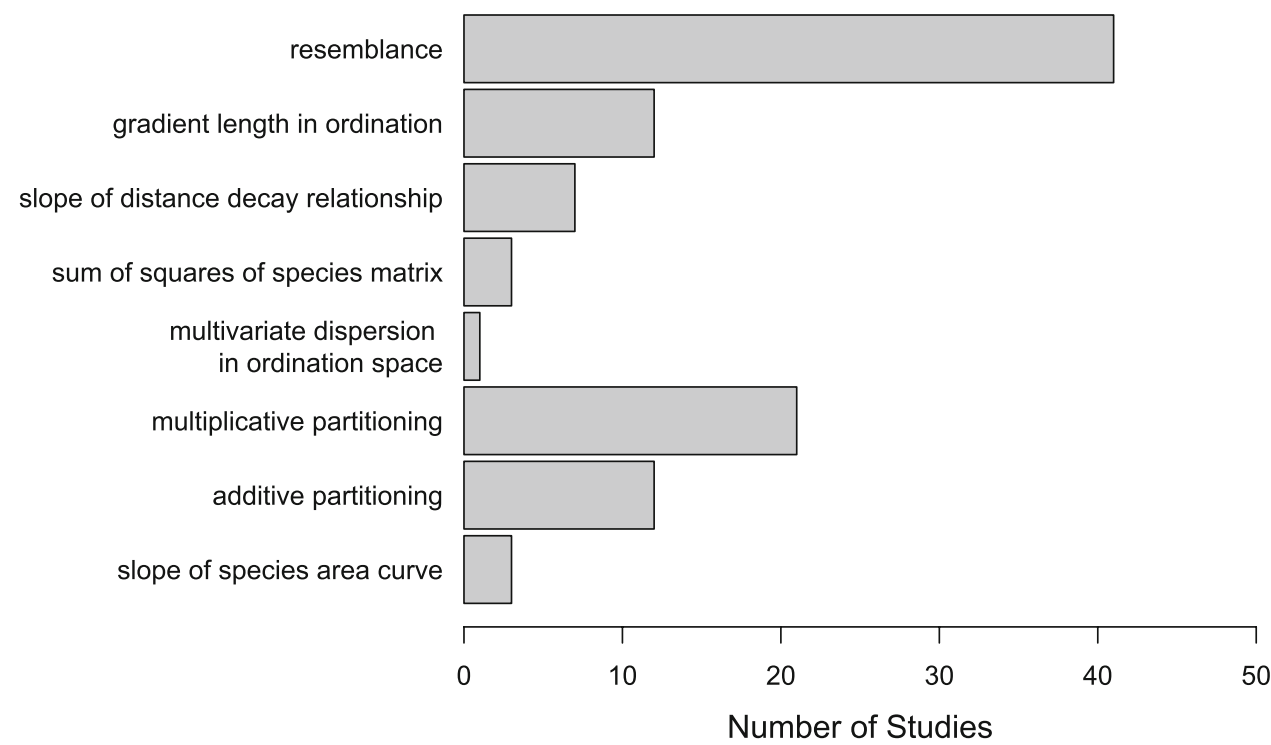

$\beta=\frac{\gamma}{\alpha}$

The reciprocal value of this coefficient can be directly explained as the proportion of species richness found in an average sample, and it thus indirectly measures similarity in species composition. This value generally decreases with the heterogeneity of the samples, but also depends on plot number and size in relation to the investigated area. It approaches $1 / n$ if the single plots share no species at all, and 1 if all plots have identical species compositions. However, for any value between these extremes, the origin of the heterogeneity between the plots cannot be distinguished. The index may yield the same value if only one plot in a data set differs completely from all others and in a situation where the data set consists of plots of moderate similarity.

Additive partitioning Recently, Veech et al. (2002) published a paper reviewing an additive notion of beta diversity closely related to Whittaker's $(1960,1972)$ multiplicative concept. They refer to Lande (1996), who proposed partitioning gamma diversity into additively combined components of alpha and beta diversity (Eq. 2). Lande (1996) was the first to use Whittaker's (1960, 1972) terminology (alpha, beta, gamma) in this context, but the conceptual idea of partitioning species diversity into additive components is much older (MacArthur et al. 1966; Levins 1968). "Additive partitioning" defines beta diversity as the average amount of diversity that is not found in a single, randomly chosen sample (Veech et al. 2002; Couteron and Pelissier 2004). However, it "does not explicitly recognize differences among samples or communities, which, after all, is the original intent of beta diversity" (Veech et al. 2002).

$\beta=\gamma-\alpha$

Concepts of beta diversity: Group 2-examining

the variation in species composition

Resemblance expressed by similarity/dissimilarity coefficients Whittaker $(1956,1960)$ suggested the use of available indices of compositional similarity, such as coefficient of community (Jaccard 1901), coincidence index (Sørensen 1948), or percentage difference (Bray and Curtis 1957), for measuring beta diversity. This notion of beta diversity is most widespread today (see Fig. 2). Therefore, a multitude of coefficients is available. Several comparative reviews have tested features and performance of (dis)similarity and distance coefficients (e.g., Cheetham and Hazel 1969; Janson and Vegelius 1981; Wolda 1981; Hubalek 1982; Shi 1993; Koleff et al. 2003a; Clarke et al. 2006).

Two relatively recent and interesting approaches are worth mentioning. Chao et al. (2005) propose a probabilistic extension to the existing coefficients of Jaccard (1901) and Sørensen (1948) to account for "unseen" shared species. A similar approach is that of Plotkin and Muller-Landau (2002) for a Sørensen-type similarity index for abundance counts, which relies on a gamma distribution to characterize "real" species-abundance structure.

Condit et al. (2002; see also Chave and Leigh 2002) propose the use of the co-dominance index of Leigh et al. (1993) as a similarity measure. It describes the probability of joint occurrences of species in compared sampling units 
(Palmer 2005); in other words, the probability that two randomly chosen individuals a distance $r$ apart belong to the same species (Chave and Leigh 2002).

Slope of the distance decay relationship This approach was formulated by Whittaker (1960) as well. It has been sparsely used since but was brought back into discussion recently by, e.g., Condit et al. (2002) and Qian et al. (2005). Distance decay is the phenomenon of decreasing similarity with increasing geographical distance (Tobler 1970, see also Qian et al. 1998; Nekola and White 1999; Tuomisto et al. 2003). The steeper the slope of the distance decay relationship, the faster species are replaced within space. Closely related to this is the suggestion of Beals (1984), to plot similarity against environmental distance (expressed by an elevational gradient). This leads to specific curvatures depending on the coefficient used, and Beals (1984) suggests that the curvature can be interpreted directly as a function of the length of the environmental gradient, which he calls beta diversity.

Sum of squares of a species matrix In a recent paper, Legendre et al. (2005) argue that the variance of a community composition table is a measure of beta diversity. They show that the total beta diversity of a data set can be derived either from the dissimilarity matrix or from the original species matrix. The authors argue that the "raw data approach" provides more statistical power and should be preferred when the variation in species composition among samples is addressed, especially when the influence of environmental drivers is being considered.

Gradient length in ordination space Whittaker was the first to suggest the use of "half-changes (HC)" as a measure of beta diversity to overcome the problem that the similarity of two samples from different ends of a gradient often equals zero, as they tend to have no species in common (Whittaker 1956, p. 321; Whittaker 1960, p. 39). Therefore, Whittaker (1956) suggested that percentage similarity should be calculated between successive plots along a gradient. At the point where the percentage similarity drops to $50 \%$, the procedure is stopped, and it is then started anew until either a new stopping point is found or the end of the gradient is reached. The number of "halfchanges" determined by this method "may thus indicate the extent of change in species populations along the gradient" (Whittaker 1956), and is therefore a measure of beta diversity (Whittaker 1960).

With increasing computing power, ordination techniques have gained in importance, and distance in ordination space is used as a proxy for beta diversity. Gauch (1973) defined " $Z$ units" of species turnover as the "axis length (100) divided by the average standard deviation of species distributions," which were later renamed "sd units" by Hill and Gauch (1980). Closely related is the concept of the mean range of species " $R$ " (Minchin 1987). A simple and direct measure of the turnover is the gradient length in detrended correspondence analysis (DCA), because it directly scales the axes in sd units. Based on a critique of available measures of floristic resemblance, Økland (1986) also proposes to use DCA axis length as a measure of beta diversity. In a slightly different approach, Ohmann and Spies (1998) used the total variation (TV) from stepwise constrained correspondence analysis (CCA) as a measure of beta diversity. Following a similar line of thought, Anderson et al. (2006) proposed the multivariate dispersion in ordination space as a measure of beta diversity. Multivariate dispersion is calculated "as the average distance (or dissimilarity) from an individual sample to the group centroid" of a (dis)similarity matrix. This has been implemented only once, in this very study (see Fig. 2), and has therefore been omitted from Table 1.

\section{Discussion}

Alpha and gamma diversity

Some authors have been critical of the fact that alpha and gamma diversity do not differ in their characteristics, only in the spatial extent over which the data are recorded. Beierkuhnlein (2001) termed both "quantitative diversity" because they are based on counts of variables (e.g., species or genera). There are other problems associated with determining gamma diversity. First, it is usually derived from combining the species found within individual samples. This is rarely a true representation of the species richness in the total area, as usually only a small proportion of the area is actually sampled. Second, although Whittaker (1960) explicitly defined gamma diversity as the diversity of a landscape, the perception of an appropriate "landscape scale" is extremely variable (see "Electronic supplementary material"). Thus, it cannot be used reproducibly without further explanation regarding the actual landscape scale for which the reference is made in a specific study.

Whittaker (1977) suggested an extended classification with seven diversity levels to account for the nested hierarchy of scales. That would be even more confusing than the three levels proposed previously, and cannot solve any of the problems discussed. However, he also applied alpha and beta diversity at different scales, thus indirectly acknowledging the superfluity of gamma diversity or any other levels such as "delta" or "omega" diversity (van der Maarel 1997). 
Table 1 A new terminology for the measurement and analysis of diversity (first column)

\begin{tabular}{|c|c|c|c|}
\hline New terms & Available concepts & $\operatorname{NoS}^{\mathrm{a}}$ & Whittaker \\
\hline Inventory diversity & Species richness, Shannon, Simpson & & Alpha, gamma \\
\hline \multirow[t]{2}{*}{ Differentiation diversity } & Resemblance (compositional (dis)similarity, distance) & 43 & \\
\hline & Sum of squares of species matrix & 3 & \\
\hline \multirow[t]{2}{*}{ Turnover $^{\mathrm{b}}$} & Gradient length in ordination & 12 & \\
\hline & Slope of distance decay relationship/halving distance & 7 & Beta \\
\hline \multirow[t]{3}{*}{ Proportional diversity } & Additive partitioning & 12 & \\
\hline & Multiplicative partitioning & 21 & \\
\hline & Slope of species-area curve & 3 & \\
\hline
\end{tabular}

Existing concepts are sorted according to the proposed new terminology (second column). The third column gives the number of studies (among the 73 analyzed) in which the existing concepts were employed (only for beta concepts). The numbers do not add up to 73 because more than one concept was employed in some articles (19)

a Numbers are based on a literature review on beta diversity in the ISI Web of Science (see "Methods")

b Turnover is a subordinate category based on the calculation of differentiation diversity, so it is filed under this category. However, species distinction is then skipped and the data are aggregated. This may lead to an increase in insight, but it also results in a loss of information

\section{Beta diversity}

\section{Concepts of beta diversity: Groupl-examining the variation in species richness}

Two measures belong to the group of concepts that disregard taxon distinction during the computation of indices: Whittaker's multiplicative beta diversity (Whittaker 1967, 1972) and the additive partitioning approach (Eq. 1, Lande 1996; Veech et al. 2002). However, Kiflawi and Spencer (2004) show that both measures are directly related, as the multiplicative measure is simply a summary statistic of the relation between the additive components. Loreau (2000) claims that the additive approach has a greater potential for consistency (see also Lande 1996) and for application to multiple scales. Veech et al. (2002) argue for additive partitioning because it measures alpha, beta and gamma in the same units. This allows for an easy comparison of the contributions of alpha and gamma to total species richness across spatial or temporal scales (Summerville and Crist 2002; Crist et al. 2003; Veech et al.2002). Consequently, it is mainly implemented to study the organization of species richness across spatial scales (e.g., Wagner et al. 2000; Gering et al. 2003) and to monitor restoration success (temporal scale; e.g., Martin et al. 2005). Nonetheless, as both concepts use only average species numbers or respective diversity values, they are not suitable for testing hypotheses regarding the drivers of species composition (Loreau 2000; Crist et al. 2003).

\section{Concepts of beta diversity: Group 2-examining variation in species composition}

The most frequently applied method of measuring the variation in species composition is the calculation of similarity or distance coefficients (see Table 1). These coefficients preserve taxon distinction during the calculation because the obtained value is determined by the species' presence and absence or their relative abundances in the compared samples, respectively. To increase the performance of the indices, recently proposed coefficients include a probability term to estimate the "true" diversity of two compared plots from the whole sampled population (Chao et al. 2005). In contrast to similarity coefficients, codominance (Condit et al. 2002, see also Leigh et al. 1993; Chave and Leigh 2002) is not a statistically valid index of similarity, because for two identical assemblages with many species, $F$ tends to zero (Chao et al. 2005). Moreover, two identical assemblages may result in different values of $F$, depending on species richness and relative abundance patterns. It is possible, however, to normalize $F$ to produce a valid similarity index. Additionally, the codominance index depends "disproportionately on the more common species, whereas turnover may be more rapid among rare species" (Pitman et al. 2001). The co-dominance index implicitly takes the geographic distance between samples into account. Therefore, it is not a measure of differentiation between sampling units but a measure of spatial organization of species in the ecosystem.

Analyses based on resemblance measures Similarity (or distance) is usually calculated between a pair of samples, but Whittaker $(1960,1972)$ proposed the use of the mean similarity calculated between all samples as a measure of beta diversity. Legendre et al. (2005) emphasize this, but simultaneously stress that the variance of the similarities is not a measure of beta diversity. There was no solution to this problem until Diserud and Ødegaard (2007) and Baselga et al. (2007) recently proposed multi-plot similarity measures that allow for the simultaneous calculation 
of similarity between multiple samples. Another approach to the calculation of multi-plot similarity (including tests of statistical significance) has been incorporated by the authors into the $R$ package simba (Jurasinski 2007), which is publicly available.

Regarding the calculation of an average similarity from single similarities between pairs of samples, we would like to add that neither the mean nor the variance take species distinction into account. In both cases, indices calculated from the raw data are aggregated at a higher level to examine heterogeneity. Both methods of aggregation neglect the phenomenon of distance decay. Because the similarity of objects is likely to decrease with distance (Tobler 1970), it is not clear how much of the variation is explained by geographical distance and how much by environmental difference. The alternative is to take the geographical distance between samples into account explicitly (Condit et al. 2002; Qian et al. 2005), but species distinction is still neglected.

It appears more promising to use the slope of the distance decay relationship directly as a measure of beta diversity, as it explicitly incorporates geographical space. However, it depends on the properties of the similarity coefficient and on the regression model used. There is no general agreement regarding the best-fitting (linear) regression model: in large-scale studies, the regression of the logarithmic similarity against geographical distance best described the relationship (Qian et al. 1998; Nekola and White 1999; Qian et al. 2005), whereas in mediumscale studies in the tropics, the best fit was achieved when using untransformed similarity and log-distance (Condit et al. 2002; Duivenvoorden et al. 2002; Phillips et al. 2003). In a recent small-scale study (unpublished), we found that the best-fitting model changes with scale. Furthermore, the best regression model often depends on the ecosystem and organisms under study (Soininen et al. 2007). Additionally, regression coefficients of the models are usually relatively poor (Jones et al. 2006).

To solve the difficulties associated with different regression models, Soininen et al. (2007) propose using the "halving distance" instead of the slope of the distance decay relationship. The halving distance is defined as the geographical distance at which the initial similarity $S$ reaches $S / 2$. The halving distance can be constructed independently from the regression model and therefore allows for comparisons across organisms and ecosystems. This method is scale-dependent, as an initial similarity must be defined rather arbitrarily (Soininen et al. 2007). Thus, results based on the slopes of distance decay relationships or the halving distance should be evaluated with care, and with the goodness of fit and the appropriate scale in mind. Nevertheless, it has relevant applications in nature conservation (Ferrier et al. 2002; Wiersma and Urban
2005), as well as in investigations of pattern and process in ecological communities (e.g., Podani et al. 1993; Garcillán and Ezcurra 2003; McDonald et al. 2005) and community assembly (dispersal versus niche; Condit et al. 2002; Gilbert and Lechowicz 2004; Jones et al. 2006).

The similarity-distance function might predict the slope of a power-law species-area curve (Condit et al. 2002). Based on this characteristic, the authors conclude that it is an appropriate measure of beta diversity. MacArthur (1965) proposed using species-area curves as an analytical tool for diversity, using the intercept of the curve as a measure of alpha diversity and the slope parameter as a measure of beta diversity (see also Caswell and Cohen 1993; Ricotta et al. 2002). However, Connor and McCoy (1979) show that this is mathematically invalid, because the slope and intercept of the power function are interdependent parameters. Additionally, there is empirical critique: even for simple systems, some component of the slope is probably due to the within-habitat diversity, so the slope cannot serve as a measure of beta diversity. Furthermore, the true area of the community being sampled must be known (Veech et al. 2002).

The suggestion of Legendre et al. (2005), that beta diversity can be measured as the sum of squares of the original species matrix, provides a more direct way of assessing the variation in species composition. They argue that canonical partitioning should be used rather than Mantel tests of dissimilarity matrices for "partitioning the spatial variation of community composition data among environmental and spatial components, and for testing hypotheses about the origin and maintenance of variation in community composition among sites." However, there is an interesting recent debate about this problem, and we agree with Tuomisto and Ruokolainen (2006) that the Mantel approach also has a niche where it is most appropriate (level 3 questions in their paper).

The use of the length of the ordination gradient as a proxy for beta diversity implies that it is indirectly based on similarity indices as well, because ordinations represent either intrinsic distances [e.g., Euclidian distance in principal components analysis (PCA) or chi-square distance in correspondence analysis (CA)—see, e.g., Legendre and Legendre 1998] or a specifically chosen distance or (dis)similarity coefficient in low-dimensional space, as in nonmetric dimensional scaling (NMDS). Because ordinations include the whole gradient within a data set, they at least partly solve the problem of zero similarity between extreme plots (De'ath 1999). Although DCA results depend on the detrending method applied (Legendre and Legendre 1998), the appealing feature of DCA scores is clearly the scaling in standard deviations, which facilitates the comparison of results between different data sets. Such standard deviations are analogs to turnover measured along 
a gradient (slope of the distance decay relationship of halving distance) for whole nonlinear data sets. Therefore, the dimensionality reduction obtained in ordination is a powerful tool for extracting gradients and reducing data complexity, and thus estimating the variation in species composition. Such methods have been frequently and successfully applied by numerous authors (e.g., Økland 1990; Pitkänen 2000; Svenning et al. 2004).

\section{A new, more descriptive terminology}

A clear and well-defined terminology, which researchers agree upon, is an important basis for intensive discussions within any field of research (Kuhn 1976; Loehle 1987). In 1984, Beals criticized Whittaker (1967) for creating the vague terms "direct gradient analysis" for ordination by environmental factors and "indirect gradient analysis" for ordination by sociological factors, and called for more descriptive terms such as "environmental ordination" and "sociological ordination." Nevertheless, the vague terms made it into the vegetation ecological terminology. Similarly, the abstract terms "alpha diversity," "beta diversity," and "gamma diversity" have became part of the biogeographical terminology, although they refer to differences in scale rather than to different data characteristics or conceptual ideas.

Here, we propose descriptive terms that better reflect the different data characteristics and conceptual ideas standing behind the various concepts and expressions of species diversity. We know that many researchers and students in ecology and related fields are aware of the Greek letters and have gained an intuitive understanding of their general meaning. With a sample being a collection of data from a plot or site, alpha diversity represents the diversity within a sample (Fig. 1). In contrast, gamma diversity is the diversity within a set of samples or at a larger scale. Usually data from the sample scale are combined and treated as one large sample to calculate the gamma diversity. Beta diversity generally reflects diversity among samples. However, as we have shown above, beta diversity has at least two distinct forms (see Fig. 1), whereas alpha and gamma diversity do not differ in their characteristics but in their relative scales. This double meaning and the duality of beta diversity can be replaced by a more meaningful and unambiguous terminology.

We do not share the opinion that beta diversity should be further used in a broad sense (Veech et al. 2002). It is not helpful to apply the term beta diversity to all concepts that somehow investigate the variation in species composition, because this requires every scientist to sort out which meaning of beta diversity the author(s) are using. However, we do agree with Veech et al. (2002) that beta diversity should not be seen "just as change along an environmental gradient." Therefore, we propose an alternative terminology to avoid confusion and to clarify the concepts. Three primary levels of diversity can be distinguished in this framework (see also Table 1): "inventory diversity," "differentiation diversity" and "proportional diversity." The variants and approaches discussed above fit into these levels as described below (see also Table 1).

Inventory diversity largely refers to Whittaker's alpha and gamma diversity, and should be used for the species data assessed within sampling units (abundance, frequency or presence/absence). It makes no difference whether these are plots, investigation areas, or geographical regions. Further, it does not matter whether inventory diversity is expressed as recorded species richness in the field or as "true" species richness estimated from the sampled data (Colwell and Coddington 1994; Chao et al. 2005), and it does not matter on which scale inventory diversity is assessed [alpha or gamma diversity in the sense of Whittaker (1960, 1972)]: it remains inventory diversity. Because of the importance of scale (Dale et al. 2002; Legendre et al. 2002; Rahbek 2005), the term "inventory diversity" should always be used with reference to the scale at which it was recorded. Nevertheless, its characteristics remain the same whilst the spatial and temporal references may change. In the conventional use of gamma diversity with an implicit reference to "landscape scale" or "global scale," it has always been necessary to clarify the exact geographical extent to which reference is made. Therefore, it may also be possible to directly refer to a specific scale of inventory diversity.

Differentiation diversity results from the comparison of samples whilst taking species composition (frequency, abundance or presence/absence) into account. Again, it does not matter which spatial or temporal scale is regarded, although essentially differentiation diversity should only be evaluated within scale levels, because inventory diversity changes with spatial extent (species-area relationship). Recent studies which emphasize the importance of beta diversity often use this "resemblance" notion (e.g., Condit et al. 2002; Srivastava 2002; Gering et al. 2003; Olden et al. 2006).

Typically, differentiation diversity can be assessed by (dis)similarity indices or measures based on such indices that are calculated between pairs of samples and, due to recent propositions (Baselga et al. 2007; Diserud and Ødegaard 2007; Jurasinski et al., unpubl.), also between multiple samples simultaneously. Computational routines for the calculation of multi-plot similarity coefficients are already available (e.g., Jurasinski 2007). Furthermore, the sum of squares of a species matrix provides a global measure of differentiation diversity (Legendre et al. 2005). Compositional similarity is thought to be determined by 
ecological processes. Thus, compositional similarity (or differentiation diversity) can be used to study the drivers of species composition.

The variation of species composition in space is a major concern in vegetation ecology and biogeography. Sometimes measurements of differentiation diversity are pooled to express a mean differentiation diversity for an area (Lennon et al. 2001; Koleff et al. 2003b; Anderson et al. 2006). However, this approach neglects distance decay. The slope of the distance decay relationship addresses this issue. It allows for an investigation of the spatial organization of differentiation diversity. Therefore, it is related to the spatial heterogeneity of species composition and is a true measurement of turnover. So why not subsume methods which compare differentiation diversity along geographical gradients under the term "turnover?"

The slope of the distance decay relationship or the "halving distance" are excellent tools for investigating spatial patterns in species distribution and their drivers. Similarly, ordination methods depend on underlying similarity or distance coefficients. In his original paper, Hill and Gauch (1980) already stresses that DCA offers a unique possibility for measuring species turnover. Thus, gradient length in ordination space is also a measurement of turnover. Within the proposed concept, turnover is always determined in a subsequent analysis of differentiation diversity describing its spatial or temporal organization (see Table 1).

Proportional diversity does not account for species differentiation but expresses the relative distribution of inventory diversity across spatial and temporal scales. The concepts of multiplicative (Whittaker 1960) and additive partitioning (Lande 1996; Loreau 2000; Veech et al. 2002) both express proportional diversity; they just differ in their mathematical approaches and are more related than it seems from first sight (Kiflawi and Spencer 2004). The term "proportional diversity" has already been used by Bambach et al. (2002) with a similar meaning, and we think that it expresses the concept quite clearly.

In the rare cases where exactly two samples make up the whole set of samples, proportional diversity may come close to being a measure of differentiation diversity (depending on the coefficient used to calculate the compositional similarity). However, as long as the species distinction is taken into account during the computation (shared and unshared species are detected and counted), we refer to the measure as a coefficient of differentiation diversity. The additive partitioning of inventory diversity into the average diversity found within scales and the average difference in diversity between scales is the appropriate technique for evaluating the distribution of inventory diversity across scales, and thus is a measure of proportional diversity as well.

\section{Conclusion}

Whittaker's (1960) biodiversity concept has heavily influenced developments in ecology. However, the term "beta diversity" in particular has been vaguely defined from the very beginning and is a rather confusing concept. This terminological ambiguity is an obstacle to development in all fields requiring more than inventory data ["alpha diversity" or "gamma diversity," sensu Whittaker (1960)]. Compositional (dis)similarity between samples ("differentiation diversity") and the variation of "inventory diversity" across scales ("proportional diversity") are important fields for future research which should not be neglected due to unclear concepts. Thus, we hope to contribute to the discussion by offering a clear terminology as a basis for scientific communication. The proposed terminology embraces all facets of the original Whittaker concepts but has a different focus. Whereas Whittaker concentrates on scale, our proposition centers on differences in data characteristics. Thus, it might be a useful conceptual complement to the alpha, beta, and gamma diversity concept.

We would like to emphasize, along with Tuomisto and Ruokolainen (2006), who "urge[d] ecologists to become more aware of the levels of abstraction in ecological questions," that increased appreciation of terminological and abstraction issues can lead to better interpretations and progress in ecology. We are aware that the now traditional terms alpha, beta, and gamma diversity will not be replaced immediately, because researchers and students have an intuitive understanding of their meaning. Nevertheless, we do think that the proposed terminology can be of value in teaching and in research regarding the communication of multiple aspects of diversity and their drivers.

Acknowledgments We gratefully acknowledge the work of R.H. Whittaker and would like to thank our colleagues Jürgen Kreyling and Anke Jentsch for fruitful discussions. Various comments from three anonymous reviewers about an earlier version greatly improved the manuscript. This contribution originates from a project of the DFG (Be 2192/5-1,2,3), which provided funding to Carl Beierkuhnlein and Gerald Jurasinski.

\section{References}

Allan JD (1975) Components of diversity. Oecologia 18:359-367 Anderson MJ, Ellingsen KE, McArdle BH (2006) Multivariate dispersion as a measure of beta diversity. Ecol Lett 9:683-693

Bambach RK, Knoll AH, Sepkoski JJ (2002) Anatomical and ecological constraints on Phanerozoic animal diversity in the marine realm. Proc Natl Acad Sci USA 99:6854-6859

Baselga A, Jimenez-Valverde A, Gilles N (2007) A multiple-site similarity measure independent of richness. Biol Lett 3:642-645

Beals EW (1984) Bray-Curtis ordination: an effective strategy for analysis of multivariate ecological data. Adv Ecol Res 14:1-55 
Beierkuhnlein C (2000) Comparing biodiversity and landscape heterogeneity at different scales. In: Brandt J, Tress B, Tress G (eds) Multifunctional landscapes. Centre for Landscape Research, Roskilde, p 214

Beierkuhnlein C (2001) Die Vielfalt der Vielfalt-Ein Vorschlag zur konzeptionellen Klärung der Biodiversität. Berichte der Reinhold-Tüxen-Gesellschaft 13:103-118

Beierkuhnlein C (2006) Biogeographie. Ulmer, Stuttgart

Bray JR, Curtis JT (1957) An ordination of the upland forest communities in Southern Wisconsin. Ecol Monogr 27:325-349

Buhk C, Retzer V, Beierkuhnlein C, Jentsch A (2007) Predicting plant species richness and vegetation patterns in cultural landscapes using disturbance parameters. Agri Ecosyst Environ 122:446452

Caswell H, Cohen JE (1993) Local and regional regulation of species-area relations: a patch-occupancy model. In: Ricklefs RE, Schluter D (eds) Species diversity in ecological communities. University of Chicago Press, Chicago, pp 99-107

Chao A (2005) Species richness estimation. In: Balakrishnan N, Read CB, Vidakovic B (eds) Encyclopedia of statistical sciences. Wiley, New York, pp 7909-7916

Chao A, Chazdon RL, Colwell RK, Shen T-J (2005) A new statistical approach for assessing similarity of species composition with incidence and abundance data. Ecol Lett 8:148-159

Chave J (2004) Neutral theory and community ecology. Ecol Lett 7:241-253

Chave J, Leigh EG (2002) A spatially explicit neutral model of $\beta$-diversity in tropical forests. Theor Popul Biol 62:153-168

Cheetham AH, Hazel JE (1969) Binary (presence-absence) similarity coefficients. J Paleontol 43:1130-1136

Chust G, Chave J, Condit R, Aguilar S, Lao S, Pérez R (2006) Determinants and spatial modeling of tree beta-diversity in a tropical forest landscape in Panama. J Veg Sci 17:83-92

Clarke KR, Somerfield PJ, Chapman MG (2006) On resemblance measures for ecological studies, including taxonomic dissimilarities and a zero-adjusted Bray-Curtis coefficient for denuded assemblages. J Exp Mar Biol Ecol 330:55-80

Colwell RK, Coddington JA (1994) Estimating terrestrial biodiversity through extrapolation. Philos Trans R Soc Lond B Biol Sci 345:101-118

Condit R et al. (2002) Beta-diversity in tropical forest trees. Science 295:666-669

Connor EF, McCoy ED (1979) The statistics and biology of the species-area relationship. Am Nat 113:791-833

Couteron P, Pelissier R (2004) Additive apportioning of species diversity: towards more sophisticated models and analyses. Oikos 107:215-221

Crist TO, Veech JA, Gering JC, Summerville KS (2003) Partitioning species diversity across landscapes and regions: a hierarchical analysis of alpha, beta, and gamma diversity. Am Nat 162:734 743

Dale MRT, Dixon P, Fortin M-J, Legendre P, Myers DE, Rosenberg MS (2002) Conceptual and mathematical relationships among methods for spatial analysis. Ecography 25:558-577

De'ath G (1999) Extended dissimilarity: a method of robust estimation of ecological distances from high beta diversity data. Plant Ecol 144:191-199

Diserud OH, Ødegaard F (2007) A multiple-site similarity measure. Biol Lett 3:20-22

Duivenvoorden JF, Svenning JC, Wright SJ (2002) Beta-diversity in tropical forests-response. Science 297:636-637

Ferrier S, Drielsma M, Manion G, Watson G (2002) Extended statistical approaches to modelling spatial pattern in biodiversity in northeast New South Wales. II. Community-level modelling. Biodivers Conserv 11:2309-2338
Fisher RA, Corbet AS, Williams CB (1943) The relationship between the number of species and the number of individuals in a random sample of an animal population. J Anim Ecol 12:42-58

Garcillán PP, Ezcurra E (2003) Biogeographic regions and betadiversity of woody dryland legumes in the Baja California peninsula. J Veg Sci 14:859-868

Gauch HG Jr (1973) The relationship between sample similarity and ecological distance. Ecology 54:618-622

Gering JC, Crist TO, Veech JA (2003) Additive partitioning of species diversity across multiple spatial scales: implications for regional conservation of biodiversity. Conserv Biol 17:488-499

Ghilarov A (1996) What does "biodiversity" mean-scientific problem or convenient myth? Trends Ecol Evol 11(7):304-306

Gilbert B, Lechowicz MJ (2004) Neutrality, niches, and dispersal in a temperate forest understory. Proc Natl Acad Sci USA 101:76517656

Gotelli NJ, Colwell RK (2001) Quantifying biodiversity: procedures and pitfalls in the measurement and comparison of species richness. Ecol Lett 4:379-391

Harrison S, Ross SJ, Lawton JH (1992) Beta diversity on geographic gradients in Britain. J Anim Ecol 61:151-158

Hector A, Bagchi R (2007) Biodiversity and ecosystem multifunctionality. Nature 448:188-190

Hill MO, Gauch HG (1980) Detrended correspondence analysis: an improved ordination technique. Vegetatio 42:47-58

Hubalek Z (1982) Coefficients of association and similarity, based on binary (presence-absence) data: an evaluation. Biol Rev Camb Philos Soc 57:669-689

Jaccard P (1901) Etude comparative de la distribution florale d'une portion des Alpes et du Jura. Bull Soc Vaudoise Sci Nat 37:547579

Janson S, Vegelius J (1981) Measures of ecological association. Oecologia 49:371-376

Jones MM, Tuomisto H, Clark DB, Olivas P (2006) Effects of mesoscale environmental heterogeneity and dispersal limitation on floristic variation in rain forest ferns. J Ecol 94:181-195

Jurasinski G (2007) simba: Similarity analysis for vegetation ecology version 0.2-5. http://cran.r-project.org/web/packages/simba/ index.html, last accessed 13 Oct 2008

Jurasinski G, Beierkuhnlein C (2006) Spatial patterns of biodiversity: assessing vegetation using hexagonal grids. Proc R Ir Acad Biol Environ 106B:401-411

Jurasinski G, Kreyling J (2007) Upward shift of alpine plants increases floristic similarity of mountain summits. J Veg Sci 18:711-718

Kiflawi M, Spencer M (2004) Confidence intervals and hypothesis testing for beta diversity. Ecology 85:2895-2900

Kluth C, Bruelheide H (2004) Using standardized sampling designs from population ecology to assess biodiversity patterns of therophyte vegetation across scales. J Biogeogr 31:363-377

Koleff P, Gaston KJ, Lennon JJ (2003a) Measuring beta diversity for presence-absence data. J Anim Ecol 72:367-382

Koleff P, Lennon JJ, Gaston KJ (2003b) Are there latitudinal gradients in species turnover? Glob Ecol Biogeogr 12:483-498

Kuhn TS (1976) Die Struktur wissenschaftlicher Revolutionen, 2nd edn. Suhrkamp, Frankfurt am Main

Lande R (1996) Statistics and partitioning of species diversity and similarity along multiple communities. Oikos 76:25-39

Legendre P, Borcard D, Peres-Neto PR (2005) Analyzing beta diversity: partitioning the spatial variation of community composition data. Ecol Monogr 75:435-450

Legendre P, Dale MRT, Fortin MJ, Gurevitch J, Hohn M, Myers D (2002) The consequences of spatial structure for the design and analysis of ecological field surveys. Ecography 25:601-615

Legendre P, Legendre L (1998) Numerical ecology. Elsevier, Amsterdam 
Leigh EG, Wright SJ, Putz FE, Herre EA (1993) The decline of tree diversity on newly isolated tropical islands: a test of a null hypothesis and some implications. Evol Ecol 7:76-102

Lennon JJ, Koleff P, Greenwood JJD, Gaston KJ (2001) The geographical structure of British bird distributions: diversity, spatial turnover and scale. J Anim Ecol 70:966-979

Levins R (1968) Evolution in changing environments. some theoretical explorations. Princeton University Press, Princeton

Loehle C (1987) Hypothesis testing in ecology: psychological aspects and the importance of theory maturation. Q Rev Biol 62:397-409

Lorance P, Souissi S, Uiblein F (2002) Point, alpha and beta diversity of carnivorous fish along a depth gradient. Aquat Living Resour $15: 263-271$

Loreau M (2000) Are communities saturated? On the relationship between $\alpha, \beta$, and $\gamma$ diversity. Ecol Lett 3:73-76

MacArthur RH (1965) Patterns of species diversity. Biol Rev Camb Philo Soc 40:510-533

MacArthur RH, Recher H, Cody ML (1966) On the relation between habitat selection and species diversity. Am Nat 100:319-332

Magurran AE (2003) Measuring biological diversity. Blackwell, Malden

Martin LM, Moloney KA, Wilsey BJ (2005) An assessment of grassland restoration success using species diversity components. J Appl Ecol 42:327-336

McDonald R et al. (2005) Species compositional similarity and ecoregions: do ecoregion boundaries represent zones of high species turnover? Biol Conserv 126:24-40

McKinney ML (2004) Measuring floristic homogenization by nonnative plants in North America. Glob Ecol Biogeogr 13:47-53

Minchin PR (1987) Simulation of multidimensional community patterns: towards a comprehensive model. Vegetatio 71:145-156

Nekola JC, White PS (1999) The distance decay of similarity in biogeography and ecology. J Biogeogr 26:867-878

Novotny V, Weiblen GD (2005) From communities to continents: beta diversity of herbivorous insects. Ann Zool Fenn 42:463-475

Ohmann JL, Spies TA (1998) Regional gradient analysis and spatial pattern of woody plant communities of Oregon forests. Ecol Monogr 68:151-182

Økland RH (1986) Rescaling of ecological gradients I-calculation of ecological distance between vegetation stands by means of their floristic composition. Nord J Bot 6:651-660

Økland RH (1990) A phytoecological study of the mire Northern Kisselbergmosen, SE Norway III-diversity and habitat niche relationships. Nord J Bot 10:191-220

Olden JD, Poff NL, McKinney ML (2006) Forecasting faunal and floral homogenization associated with human population geography in North America. Biol Conserv 127:261-271

Olden JD, Rooney TP (2006) On defining and quantifying biotic homogenization. Glob Ecol Biogeogr 15:113-120

Palmer MW (2005) Distance decay in an old-growth neotropical forest. J Veg Sci 16:161-166

Peirce CS (1884) The numerical measure of the success of predictions. Science 4:453-454

Phillips OL et al. (2003) Habitat association among Amazonian tree species: a landscape-scale approach. J Ecol 91:757-775

Pitkänen S (2000) Classification of vegetational diversity in managed boreal forests in eastern Finland. Plant Ecol 146:11-28

Pitman NCA et al. (2001) Dominance and distribution of tree species in upper Amazonian terra firme forests. Ecology 82:2101-2117

Plotkin JB, Muller-Landau HC (2002) Sampling the species composition of a landscape. Ecology 83(12):3344-3356

Podani J, Czaran T, Bartha S (1993) Pattern, area, and diversity: the importance of spatial scale in species assemblages. Abst Bota $17: 37-51$

Podani J (2006) With a machete through the djungle: some thoughts on community diversity. Acta Biotheor 54:125-131
Preston FW (1948) The commonness, and rarity, of species. Ecology 29:254-283

Qian H, Klinka K, Kayahara GJ (1998) Longitudinal patterns of plant diversity in the North American boreal forest. Plant Ecol V138:161-178

Qian H, Ricklefs RE, White PS (2005) Beta diversity of angiosperms in temperate floras of eastern Asia and eastern North America. Ecol Lett 8:15-22

Rahbek C (2005) The role of spatial scale and the perception of largescale species-richness patterns. Ecol Lett 8:224-239

Ricotta C (2005) Through the djungle of biological diversity. Acta Biotheo 53:29-38

Ricotta C, Carranza ML, Avena G (2002) Computing beta-diversity from species-area curves. Basic Appl Ecol 3:15-18

Rosenzweig ML (1995) Species diversity in space and time. Cambridge University Press, London

Ruokolainen K, Linna A, Tuomisto H (1997) Use of Melastomataceae and Pteridophytes for revealing phytogeographical patterns in Amazonian rain forests. J Trop Ecol 13:243-256

Sarkar S (2005) Biodiversity and environmental philosophy: an introduction (Cambridge Studies in Philosophy and Biology). Cambridge University Press, New York

Sarkar S (2006) Ecological diversity and biodiversity as concepts for conservation planning: comments on Ricotta. Acta Biotheor 54:133-140

Shannon CE, Weaver W (1949) The mathematical theory of communication. University Illinois Press, Urbana

Shi GR (1993) Multivariate data analysis in palaeoecology and palaeobiogeography-a review. Palaeogeogr Palaeoclimatol Palaeoecol 105:199-234

Simpson EH (1949) The measurement of diversity. Nature 163:688

Soininen J, McDonald R, Hillebrand H (2007) The distance decay of similarity in ecological communities. Ecography 30:3-12

Sørensen T (1948) A method of establishing groups of equal amplitude in plant sociology based on similarity of species content. Biologiske Skrifter 5:1-34

Srivastava DS (2002) The role of conservation in expanding biodiversity research. Oikos 98:351-360

Steinitz O, Heller J, Tsoar A, Rotem D, Kadmon R (2005) Predicting regional patterns of similarity in species composition for conservation planning. Conserv Biol 19:1978-1988

Summerville KS, Crist TO (2002) Effects of timber harvest on forest Lepidoptera: community, guild, and species responses. Ecol Appl 12:820-835

Svenning JC, Kinner DA, Stallard RF, Engelbrecht BMJ, Wright SJ (2004) Ecological determinism in plant community structure across a tropical forest landscape. Ecology 85:25262538

Tobler WR (1970) A computer movie simulating urban growth in the Detroit region. Econ Geogr 46:234-240

Tuomisto H, Ruokolainen K (2006) Analyzing or explaining betadiversity? Understanding the targets of different methods of analysis. Ecology 87:2697-2708

Tuomisto H, Ruokolainen K, Yli-Halla M (2003) Dispersal, environment, and floristic variation of Western Amazonian Forests. Science 299:241-244

van der Maarel E (1997) Biodiversity: from babel to biosphere management. Opulus Press, Uppsala

Veech JA, Summerville KS, Crist TO, Gering JC (2002) The additive partitioning of species diversity: recent revival of an old idea. Oikos 99:3-9

Vellend M (2001) Do commonly used indices of beta-diversity measure species turnover? J Veg Sci 12:545-552

Wagner HH, Wildi O, Ewald KC (2000) Additive partitioning of plants species diversity in an agricultural mosaic landscape. Landsc Ecol 15:219-227 
Whittaker RH (1956) Vegetation of the Great Smoky Mountains. Ecol Monogr 26:1-80

Whittaker RH (1960) Vegetation of the Siskiyou Mountains, Oregon and California. Ecol Monogr 30:279-338

Whittaker RH (1967) Gradient analysis of vegetation. Biol Rev Camb Philos Soc 42:207-246

Whittaker RH (1972) Evolution and measurement of species diversity. Taxon 12:213-251

Whittaker RH (1977) Evolution of species diversity in land communities. Evol Biol 10:1
Wiersma YF, Urban DL (2005) Beta diversity and nature reserve system design in the Yukon, Canada. Conserv Biol 19:12621272

Williams CB (1950) The application of the logarithmic series to the frequency of occurrence of plant species in quadrats. $\mathrm{J}$ Ecol $38: 107-138$

Wolda H (1981) Similarity indices, sample size and diversity. Oecologia 50:296-302 